\title{
Studies of discrete symmetries in a purely leptonic system using the Jagiellonian Positron Emission Tomograph
}

P. Moskal ${ }^{1, \star}$, D. Alfs ${ }^{1}$, T. Bednarski ${ }^{1}$, P. Białas ${ }^{1}$, C. Curceanu ${ }^{2}$, E. Czerwiński ${ }^{1}$, K. Dulski $^{1}$, A. Gajos ${ }^{1}$, B. Głowacz ${ }^{1}$, N. Gupta-Sharma ${ }^{1}$, M. Gorgol $^{3}$, B. C. Hiesmayr ${ }^{4}$, B. Jasińska ${ }^{3}$, D. Kamińska ${ }^{1}$, O. Khreptak ${ }^{1}$, G. Korcyl ${ }^{1}$, P. Kowalski ${ }^{5}$, W. Krzemień ${ }^{6}$, N. Krawczyk ${ }^{1}$, E. Kubicz ${ }^{1}$, M. Mohammed ${ }^{1}$, Sz. Niedźwiecki ${ }^{1}$, M. Pawlik-Niedźwiecka ${ }^{1}$, L. Raczyński ${ }^{5}$, Z. Rudy ${ }^{1}$, M. Silarski ${ }^{1}$, J. Smyrski ${ }^{1}$, A. Wieczorek ${ }^{1}$, W. Wiślicki ${ }^{5}$, B. Zgardzińska ${ }^{3}$, and M. Zieliński ${ }^{1}$

${ }^{1}$ Faculty of Physics, Astronomy and Applied Computer Science, Jagiellonian University, Cracow, Poland

${ }^{2}$ INFN, Laboratori Nazionali di Frascati, 00044 Frascati, Italy

${ }^{3}$ Institute of Physics, Maria Curie-Skłodowska University, 20-031 Lublin, Poland

${ }^{4}$ Faculty of Physics, University of Vienna, 1090 Vienna, Austria

${ }^{5}$ Świerk Computing Centre, National Centre for Nuclear Research, 05-400 Otwock-Świerk, Poland

${ }^{6}$ High Energy Department, National Centre for Nuclear Research, 05-400 Otwock-Świerk, Poland

\begin{abstract}
Discrete symmetries such as parity (P), charge-conjugation (C) and time reversal $(\mathrm{T})$ are of fundamental importance in physics and cosmology. Breaking of charge conjugation symmetry (C) and its combination with parity (CP) constitute necessary conditions for the existence of the asymmetry between matter and antimatter in the observed Universe. The presently known sources of discrete symmetries violations can account for only a tiny fraction of the excess of matter over antimatter. So far $\mathrm{CP}$ and $\mathrm{T}$ symmetries violations were observed only for systems involving quarks and they were never reported for the purely leptonic objects. In this article we describe briefly an experimental proposal for the test of discrete symmetries in the decays of positronium atom which is made exclusively of leptons. The experiments are conducted by means of the Jagiellonian Positron Emission Tomograph (J-PET) which is constructed from strips of plastic scintillators enabling registration of photons from the positronium annihilation. J-PET tomograph together with the positronium target system enable to measure expectation values for the discrete symmetries odd operators constructed from (i) spin vector of the ortho-positronium atom, (ii) momentum vectors of photons originating from the decay of positronium, and (iii) linear polarization direction of annihilation photons. Linearly polarized positronium will be produced in the highly porous aerogel or polymer targets, exploiting longitudinally polarized positrons emitted by the sodium ${ }^{22} \mathrm{Na}$ isotope. Information about the polarization vector of orthopositronium will be available on the event by event basis and will be reconstructed from the known position of the positron source and the reconstructed position of the orthopositronium annihilation. In 2016 the first tests and calibration runs are planned, and the data collection with high statistics will commence in the year 2017.
\end{abstract}

^e-mail:p.moskal@uj.edu.pl 


\section{Introduction}

The violation of the combined symmetries of charge conjugation and spatial parity (CP) was discovered 52 years ago in the decays of neutral $\mathrm{K}$ mesons [1]. CP symmetry is connected with the time reversal symmetry $(\mathrm{T})$ via the CPT theorem $[2,3]$. Time reversal concept is exceptionally attractive epistemologically [4] and its description is challenging [5], but nevertheless it took almost half a century till it was seen in a way independent of CP and CPT symmetries [6]. The T symmetry violation was observed in decays of the quantum entangled neutral B mesons [6, 7] using a method proposed in references $[8,9]$. At present mesons, which are systems of quark and antiquark bound via strong interaction, remain the only objects in which violations of T and CP symmetries were observed. Therefore, it appears natural to ask a question about the degree of the violation of these symmetries in systems made of leptons. An ideal object for this kind of studies is the positronium atom: a system of lepton and antilepton bound via electromagnetic interaction. A test of the discrete symmetries in the decays of positronium into photons is limited by the photon-photon interaction which is expected to mimic the symmetry violation at the level of about $10^{-9}$ only $[10,11]$. This is still about six orders of magnitude smaller than the present experimental limits equal to about $3 \times 10^{-3}[12,13]$.

In this article we briefly describe a new proposal for tests of the T, CP as well as CPT symmetries in the decays of the positronium. The experiments are conducted using the Jagiellonian Positron Emission Tomograph (J-PET). For the detailed description of the motivation to perform such experiments the interested reader is referred to the recent article [15].

\section{Experimental method}

J-PET tomograph consists of three cylindrical layers of plastic scintillator strips. The diameter of the inner layer is equal to $85 \mathrm{~cm}$ and the length of the scintillators amounts to $50 \mathrm{~cm}$. Results of the first tests of single and double module prototypes are described in references [16-19]. For further details about the data acquisition system and analysis, simulation of the detector response, as well as hittime and hit-position reconstruction methods the interested reader is referred to references [20-26]. Finally, the details about detector geometry along with its properties relevant for the registration of photons from the decays of ortho-positronium atoms are described in the recent article [27].

Ortho-positronium atoms will be produced by the positrons thermalised in the porous aerogels or polymers [28]. Positrons will be emitted by the ${ }^{22} \mathrm{Na}$ isotope positioned in the center of the cylinder covered with the porous material. Due to the parity violation by the weak interaction, positrons emitted in the decay of sodium: ${ }^{22} \mathrm{Na} \rightarrow{ }^{22} \mathrm{Ne} e^{*} e^{+} v_{e} \rightarrow{ }^{22} \mathrm{Ne} \gamma e^{+} v_{e}$, are longitudinally polarized with the average polarization equal to about $67 \%[13,14]$. This value decreases only by about $8 \%$ during the thermalisation process $[29,30]$ and enables to produce ortho-positronium atoms polarized along the direction of the positron emission with the polarization degree of about $41 \%$ when using sodium ${ }^{22} \mathrm{Na}[13,31]$. The polarization axis varies from event to event, but it can be determined from the known points of the positron emission and positronium annihilation. The annihilation place will be reconstructed based on the trilateration method [32]. The positronium target will be inserted inside the J-PET detector. Photons from the ortho-positronium decay and from the deexcitation of ${ }^{22} \mathrm{Ne}$ are detected predominantly via the Compton interaction in the scintillator strips. A typical event useful for the studies of the discrete symmetries will include four or more signals: three signals from photons originating from the decay of ortho-positronium atom, one signal from the photon emitted by the excited ${ }^{22} \mathrm{Ne}$ isotope, and possibly signals from the secondary scattering in the detectors. The measurement of energy loss of photons, their time of interaction, as well as their relative angles will allow to disentangle signals from annihilation, deexcitation and secondary photons. The measurement of the time difference between the registration of annihilation and deexcitation photons will enable to 
disentangle the annihilation of ortho-positronium from the direct electron-positron annihilation and from the annihilation of para-positronium.

In the case of the Compton scattering there is no unique relation between the energy loss and the energy of the interacting photon. Nevertheless, in the case of the registration of three photons from the ortho-positronium decay, their energies may be reconstructed based on the measurement of relative angles between the photons and the application of the energy and momentum conservation [27]. The angular resolution of the J-PET is equal to about 0.4 degree. This results in the energy resolution of about $5 \mathrm{keV}$ for the registration of photons originating from the decay of ortho-positronium [27]. Based on the tests of prototype detector with the $30 \mathrm{~cm}$ long scintillators [17, 18] and simulations [19], the expected hit-time resolution of the J-PET detector is in the range from about $100 \mathrm{ps}(\sigma)$ to about $250 \mathrm{ps}(\sigma)$ for deposited energy range from $340 \mathrm{keV}$ to $50 \mathrm{keV}$, respectively. High time and angular resolution enables to suppress by nine orders of magnitude the background due to the electron-positron annihilation into two photons followed by the secondary scatterings in the scintillator strips [27]. The secondary photon scattering in the detector can be turned into advantage since it allows to determine a linear polarization $(\vec{\epsilon})$ of the primary photon at the moment of the scattering [15].

The above brief description shows that the J-PET tomograph, equipped with the dedicated positronium target, enables to measure photons momenta, their polarizations and the spin of orthopositronium. This opens possibilities to test discrete symmetries in the decays of ortho-positronium via measurement of the expectation values of the various symmetry odd operators which may be constructed from the spin polarization vector of ortho-positronium $(\vec{S})$ as well as from momentum vectors $(\vec{k})$ and polarization directions $(\vec{\epsilon})$ of photons originating from the decay of ortho-positronium. Exemplary operators are shown in Tab. 1.

Table 1. Operators for the $o-P s \rightarrow 3 \gamma$ process and their properties with respect to the C, P, T, CP and CPT symmetries. New operators (including $\vec{\epsilon}$ ) not explored before are shown in the last three rows. The decay plane is oriented by the ordering of photons according to the descending momentum: $k_{1}>k_{2}>k_{3}$. The table is adapted from reference [15].

\begin{tabular}{|c|c|c|c|c|c|}
\hline Operator & C & P & T & CP & CPT \\
\hline$\vec{S} \cdot \overrightarrow{k_{1}}$ & + & - & + & - & - \\
\hline$\vec{S} \cdot\left(\overrightarrow{k_{1}} \times \overrightarrow{k_{2}}\right)$ & + & + & - & + & - \\
\hline$\left(\vec{S} \cdot \overrightarrow{k_{1}}\right)\left(\vec{S} \cdot\left(\overrightarrow{k_{1}} \times \overrightarrow{k_{2}}\right)\right)$ & + & - & - & - & + \\
\hline $\overrightarrow{k_{2}} \times \overrightarrow{\epsilon_{1}}$ & + & - & - & - & + \\
\hline$\vec{S} \cdot \overrightarrow{\epsilon_{1}}$ & + & + & - & + & - \\
\hline$\vec{S} \cdot\left(\overrightarrow{k_{2}} \times \overrightarrow{\epsilon_{1}}\right)$ & + & - & + & - & - \\
\hline
\end{tabular}

\section{Summary}

Jagiellonian Positron Emission Tomograph is a multi-purpose detector which enables broad interdisciplinary investigations including, among others: (i) medical imaging [19], (ii) studies of discrete symmetries in the decays of positronium atoms [15, 27], (iii) quantum entanglement of high energy photons originating from the decay of ortho-positronium, as well as (iv) research in the field of lifeand material-sciences [33-35]. In this article properties of the J-PET detector relevant for the studies of the discrete symmetries were briefly described. 


\section{References}

[1] J. H. Christenson, J. W. Cronin, V. L. Fitch, R. Turlay, Phys. Rev. Lett. 13, 138 (1964)

[2] J. S. Bell, Proc. R. Soc. London Ser. A 231, 479 (1955)

[3] G. Lüders, Ann. Phys. 2, 1 (1957); G. Lüders, Ann. Phys. (N.Y.) 281, 1004 (2000)

[4] J. Gołosz, J. Gen. Philos. Sci. (2016)

[5] T. Durt, A. Di Domenico, B. Hiesmayr, arXiv:1512.0843 (2015)

[6] J. P. Lees et al., Phys. Rev. Lett. 109, 211801 (2012)

[7] J. P. Lees et al., Phys. Rev. D 94, 011101(R) (2016)

[8] J. Bernabeu et al., JHEP 1208, 64 (2012)

[9] J. Bernabeu, A. Di Domenico, P. Villanueva-Perez, Nucl. Phys. B 868, 102 (2013)

[10] W. Bernreuther et al., Z. Phys. C 41, 143 (1988)

[11] B. K. Arbic et al., Phys. Rev. A 37, 3189 (1988)

[12] T. Yamazaki et al., Phys. Rev. Lett. 104, 083401 (2010)

[13] P. A. Vetter, S. J. Freedman, Phys. Rev. Lett. 91, 263401 (2003)

[14] P. Coleman, Positron Beams and Their Applications, World Scientific (2000)

[15] P. Moskal et al., Acta Phys. Polon. B 47, 509 (2016)

[16] P. Moskal et al., Bio-Algorithms and Med-Systems 7, 73 (2011)

[17] P. Moskal et al., Nucl. Instrum. and Meth. A 764, 317 (2014)

[18] P. Moskal et al., Nucl. Instrum. and Meth. A 775, 54 (2015)

[19] P. Moskal et al., Phys. Med. Biol. 61, 2025 (2016)

[20] L. Raczyński et al., Nucl. Instrum. and Meth. A 764, 186 (2014)

[21] L. Raczyński et al., Nucl. Instrum. and Meth. A 786, 105 (2015)

[22] N. G. Sharma et al., Nukleonika 60, 765 (2015)

[23] P. Moskal et al., Acta Phys. Polon. A 127, 1495 (2015)

[24] P. Kowalski et al., Acta Phys. Polon. B 47, 549 (2016)

[25] G. Korcyl et al., Acta Phys. Polon. B 47, 491 (2016)

[26] W. Krzemień et al., Acta Phys. Polon. B 47, 561 (2016)

[27] D. Kamińska et al., Eur. Phys. J. C 76, 445 (2016)

[28] B. Jasińska et al., Acta Phys. Polon. B 47, 453 (2016)

[29] J. Van House, P. W. Zitzewitz, Phys. Rev. A 29, 96 (1984)

[30] P. W. Zitzewitz et al., Phys. Rev. Lett. 43, 1281 (1979)

[31] J. Yang et al., Jpn. J. Appl. Phys. 36, 3764 (1997)

[32] A. Gajos et al., Nucl. Instrum. and Meth. A 819, 54 (2016)

[33] E. Kubicz et al., Nukleonika 60, 749 (2015)

[34] A. Wieczorek et al., Acta Phys. Polon. A 127, 1487 (2015)

[35] A. Wieczorek et al., Nukleonika 60, 777 (2015) 